\title{
Study of Modern Human Evolution via Comparative Analysis with the Neanderthal Genome
}

\author{
Musaddeque Ahmed, Ping Liang* \\ Department of Biological Sciences, Brock University, St. Catharines, ON L2S 3A1, Canada
}

\begin{abstract}
Many other human species appeared in evolution in the last 6 million years that have not been able to survive to modern times and are broadly known as archaic humans, as opposed to the extant modern humans. It has always been considered fascinating to compare the modern human genome with that of archaic humans to identify modern human-specific sequence variants and figure out those that made modern humans different from their predecessors or cousin species. Neanderthals are the latest humans to become extinct, and many factors made them the best representatives of archaic humans. Even though a number of comparisons have been made sporadically between Neanderthals and modern humans, mostly following a candidate gene approach, the major breakthrough took place with the sequencing of the Neanderthal genome. The initial genome-wide comparison, based on the first draft of the Neanderthal genome, has generated some interesting inferences regarding variations in functional elements that are not shared by the two species and the debated admixture question. However, there are certain other genetic elements that were not included or included at a smaller scale in those studies, and they should be compared comprehensively to better understand the molecular make-up of modern humans and their phenotypic characteristics. Besides briefly discussing the important outcomes of the comparative analyses made so far between modern humans and Neanderthals, we propose that future comparative studies may include retrotransposons, pseudogenes, and conserved non-coding regions, all of which might have played significant roles during the evolution of modern humans.
\end{abstract}

Keywords: biological evolution, comparative genomics, humans, Neanderthals

\section{Introduction}

Modern humans are indeed a very young species compared to their cousins, evolving just about 200,000 years ago (ya), which is a fraction of the 6 million years since the divergence of the human and chimpanzee lineages [1]. Fossils suggest that modern humans first emerged in East Africa and spread fairly quickly all over the world in the next 185,000 years or so (reviewed in Lu et al. [2]). After the divergence of humans and chimps, the major landmark in human history is the emergence of bipedals about 4 million years ago (mya), which enabled them to use their two feet as hands. Many species evolved afterwards until the evolution of Homo erectus, who, for the first time, migrated out of Southern Africa and initiated the spread of humans all around the globe. The migrated population of Homo erectus in East Africa eventually gave rise to modern humans about
200,000 ya and to Homo neanderthalensis, or Neanderthals, about 400,000 ya [3, 4]. Neanderthals survived until 28,000 ya, while modern humans are still surviving [5]. During the latter part of their existence, Neanderthals lived in Europe, as well as in Western Asia and the Middle East [6, 7]. Various lines of evidence suggest that modern humans started to migrate from East Africa to Europe and other parts of the world 100,000 ya, and the fossil evidence of humans and Neanderthals indicated that these species might have come into contact as early as 80,000 ya and co-habited for up to 10,000 years at certain geographic locations [6].

In the field of evolutionary biology, one of the most sought after questions has been what made modern humans superior than other related species -i.e., the genomic features that are unique to humans. The whole-genome sequencing of chimps, rhesus macaque, and other primates has given considerable boosts in this field, as the sequences of these 
primates opened up the possibility to conduct comprehensive comparative studies to the single-nucleotide level $[8,9]$. Many attempts have been taken to identify the genetic reasons why modern humans developed such complex biological features than other primates, including the larger brain-to-body ratio, bipedalism, morphological changes, and significant development of communication skills and cognitive behavior. Recent studies have used various statistical methods to compare the sequence of these primates with humans in an attempt to find human-specific genes and gene regulatory sequences, eventually showing unexpectedly rapid evolution in the human lineage after the divergence from the ancestral primates [10-15]. The results from these analyses exhibit a good overview of the human-specific genomic elements, but these results are unable to distinguish which of these human-specific elements are specific to modern humans only. Since there has been no complete genome sequence of any archaic humans until recently, such sequence comparisons have been made only between the modern human genome and other primates, bypassing archaic humans, resulting in an overwhelming number of differences and the inability to identify which sequences changes are unique to modern humans and which are shared by all Homo species. Therefore, the comparative analysis between modern humans and archaic humans is expected to be more interesting and valuable by being more effective in identifying the critical genes and/or regulatory elements that may be fully or partially responsible for the evolution of the modern humans over other humans.

In order to identify such sequence changes, the modern human genome sequence must be compared with that of archaic humans. Neanderthals have always been a desired target for this purpose for multiple reasons. They are the closest cousins of modern humans, both anatomically and based on intelligence. Their slightly larger brain and wider body structure are the primary anatomical differences from the modern humans [16]. Fossil evidence suggests that Neanderthals used stone tools, they were hunter gatherers, and they had a social life, indicating that they had similar intelligence as modern humans until about 50,000 ya, when a "Creative explosion" occurred in modern humans $[17,18]$. Another critical reason to target Neanderthals is that they are the latest archaic humans to go extinct, and their remains have been found in sufficiently good condition for analyzing down to the molecular level [19-21]. To make a comprehensive genomic comparison between modern and archaic humans, the whole genome of archaic humans has to be sequenced. The availability and widespread use of massively parallel high-throughput sequencing have now made it possible to sequence archaic genomes, which seemed impossible even a decade ago. The idea of sequencing an ancient genome was first implemented on cave bear [22] and, after its success, the mammoth genome [23]. The initial sequencing attempts on Neanderthals included sequencing of the mitochondrial genome, which was successful to a certain extent [24-26]. These successes eventually led paleogeneticists to attempt sequencing Neanderthal nuclear genomes. Almost all of the attempts have been made by amplifying the genome fragments via PCR and parallel sequencing, while some involve the use of a metagenomic approach. The early success of such attempts eventually led to the establishment of the Neanderthal Genome Project in 2005, which first announced the complete genome sequence of Neanderthals in 2010 [27] and recently released a cleaner and higher-coverage version (http://www.eva.mpg.de/nean dertal/). Comprehensive comparisons have been made by the group on certain genetic entities with some very interesting inferences, based on the initial low-coverage sequencing data, but improvements in many aspects can be made by utilizing the recent, better version of the sequence and considering more types of genetic variances in the study. Here, we will discuss the inferences made from the comparison and what can be done next to answer some interesting questions regarding the evolution of modern humans.

\section{Why Whole Genome Sequence of Nean- derthals?}

Many lines of archaeological evidence indicate that humans and Neanderthals may have coexisted in certain geographic locations. This gives rise to the most debatable question regarding the recent history of humans: did modern humans and Neanderthals interbreed? If they did, was it to an extent where meaningful exchange of genetic information may have occurred? Do we still carry any genetic elements of Neanderthals? Comparing the genome sequences is probably the best way to answer all these questions. All other features, including the level of intelligence in Neanderthals, have been speculated from bones, settlements, or artifacts found, and there is no way to be certain about the practicality or validity of the inferences made from these remains. The hypothesis that Neanderthals were able to practice complex behavior has already been disputed [28-30]. There has also been a significant amount of debate about the admixture of humans and Neanderthals. Morphological analyses have provided strong arguments both for and against the genetic exchange between these two species [31,32], as have the comparative analyses of DNA sequences of these species [33-35]. The genome sequence itself can not validate many of these inferences, either, but it can answer the question of admixture and articulate the 
genetic elements that are unique to Neanderthals or to humans or those that are shared by both. Studies on the expression or protein functions in association with these unique elements, despite being plausible only for those found in the human genome, can eventually facilitate analysis of complex biological phenomena, such as reasoning, language, or other qualitative or behavioral traits, at the molecular level.

In the past, a candidate gene approach has been successfully implemented to identify the presence or variance of certain genes that were believed to be modern humanspecific. Using this approach, a number of speculations about Neanderthals could be made, including their skin and hair color. It was also discovered that Neanderthals had the same FOXP2 gene as modern humans, which was previously linked to language ability [36]. This approach of fishing for particular genes proved efficient, but there is a lot more than known genes in the whole genome that may play a critical regulatory role in gene expression and thus development. For instance, almost half of the human genome consists of transposable elements; these elements can affect gene expression by activating or deactivation functional genetic elements and by altering the protein coding by creating alternative splicing or creating new chimeric genes ([37-39], reviewed in [40, 41]). Transposable elements are also polymorphic among different populations of modern humans, and their association with phenotypic traits, including diseases, has been extensively studied, albeit with a lot more to be learned [42-44]. The whole-genome sequence is necessary for identifying transposable element insertions that may have taken place only in modern humans and subsequently assessing their functional impact.

\section{Comparative analysis}

The only part of the Neanderthal genome that was sequenced completely from multiple specimens until recently was the mitochondrial DNA (mtDNA). The major analysis that was made with the mtDNA sequence was to seek an answer to the question of interbreeding. The Neanderthal mtDNA sequence consistently falls outside the spectrum of variations observed in the modern human mtDNA sequence, indicating no interbreeding between the species [24-26, $45,46]$. Previously, besides detecting specific mutations in $M C P H 1$ and FOXP2, the candidate gene approach also detected the presence of fragments of the MCIR gene that may indicate the red hair and pale skin of Neanderthals [47], segments of the ABO blood group locus [48], and a taste receptor gene [49]. Despite many critical technical challenges in sequencing ancient genomes ([26]; reviewed in [50]), the whole genome of Neanderthals has been sequenced recently by Green et al. [27], and it allowed this research group to perform a genome-wide sequence comparison with the modern human genome sequence, and the significant results from the initial analyses are discussed briefly below.

\section{Substitutions and indels}

Green et al. [27] inferred that 10,535,445 substitutions and 479,863 indels have occurred in the modern human genome after the divergence from chimps. The vast majority of these occurred before Neanderthals and modern humans diverged. However, 78 non-synonymous nucleotide substitutions that are fixed for a derived state in modern humans are different in the Neanderthal counterpart, as Neanderthals carry the ancestral state of these polymorphic nucleotides. Only five genes were identified that have more than one fixed substitution in their coding regions, and one of them has an altered start or stop codon. It is particularly interesting that three of these genes are expressed in skin, including RPTN, which encodes the protein repetin, and TRPMI, which encodes melastatin. This might be indicative of a change in selection of skin physiology in the modern human lineage. When looking into differences in regulatory elements, a total of 132 substitutions and 36 indels were identified in the untranslated regions. One microRNA of unknown function, hsa-mir-1304, which was identified by parallel sequencing of human embryonic stem cells [51], was found to have one fixed substitution and one single nucleotide insertion. Since the substitution occurs in the seed region, it is not unlikely that this microRNA has different targets between these two species.

\section{Selective sweeps in modern humans}

There are 212 positively selected regions identified in modern humans that occurred early during the history in conjunction with or shortly after their divergence from Neanderthals. The largest of the positively selected regions contains the gene THADA, single-nucleotide polymorphisms (SNPs) in the vicinity of which have been linked with type II diabetes [52]. This change may have affected the energy metabolism of early modern humans. A number of other genes that lie on these selectively swept regions have been associated with other genetic disorders, such as autism, schizophrenia, and Down syndrome. Since both autism and schizophrenia are related to cognitive development, it could be assumed that multiple genes involved in cognitive development in humans were positively selected early in the history of modern humans.

\section{Admixture}

Even though previous studies with Neanderthal mtDNA $[26,33]$ and initial sequencing of nuclear DNA showed no 
evidence of interbreeding between Neanderthals and humans [21, 53], the most striking revelation after the comparative analyses between the whole-genome sequence of Neanderthals and multiple modern human individual genomic sequences was the demonstration of admixture between these two species. Green et al. [27] compared the Neanderthal genome with eight modern-day human genomes of European Americans, East Asians, and West African ancestry. Surprisingly, the Neanderthal genome appeared more similar to all non-African genomes than to African ones. They share significantly more derived alleles (alleles that are different from in chimp) with non-African populations than with the African, and when compared with European and Asian individual genomes, Neanderthals are found equally close to both populations. This and some other analyses made by the group only indicate an exchange of DNA between Neanderthals and the non-African population. With further comparative analysis, the same group also identified that gene flow occurred unidirectionally from Neanderthals to the modern non-African human population.

A couple of other findings from different experiments, along with a genome-wide comparison, provide strong evidence for exchange of genetic information. The first finding involves the MAPT locus in chromosome 17, which has two distinct haplotypes, $\mathrm{H} 1$ and $\mathrm{H} 2$. $\mathrm{H} 1$ is abundant in almost all populations of modern humans, while $\mathrm{H} 2$ is found only among Europeans and found to have entered into the Homo lineage approximately only 10,000 to 30,000 ya. However, a comparison between $\mathrm{H} 1$ and $\mathrm{H} 2$ in chimp suggests that the common founder of $\mathrm{H} 1$ and $\mathrm{H} 2$ is far older than 30,000 years. Even though the Neanderthal genome sequence has been found to carry the $\mathrm{H} 1$ haplotype, coinhabitation of the $\mathrm{H} 2$ chromosome carriers during the time period when modern humans coexisted with archaic humans can not be ruled out because of the scarcity of archaic genome data. Thus, one can still argue that the $\mathrm{H} 2$ haplotype found in modern humans could possibly be a result of horizontal gene transfer between modern humans and Neanderthals and remained in modern humans under selective pressure, possibly because the $\mathrm{H} 1$ haplotype has a role in neurodegenerative diseases [54]. In a similar scenario, haplotype D of the microcephalin gene is found to have originated 1.1 mya in a lineage other than modern humans but integrated into the modern human genome only about 37,000 ya. It has thus been speculated that this haplotype was horizontally transferred into modern humans from archaic humans, most likely Neanderthals [34]. However, in a more recent study, the microcephalin locus from a Neanderthal individual in Italy was sequenced and found to be homozygous for the ancestral non-D haplotype [55]. The whole-genome study by Green et al. [27] does not support these speculations, either, since the Neanderthals they analyzed do not carry these D-haplotypes.

One striking revelation from the whole-genome comparison by Green et al. [27] is the equal level of similarity of the Neanderthal genome with Papuan and Chinese and French, although fossil records show the existence of Neanderthals only in Europe and western Asia. The group explained this anomaly by arguing that the interbreeding between the species occurred earlier than previously expected, before the divergence of Europeans, East Asians, and Papuans. Archaeological evidence suggests that modern humans appeared in the Middle East before 100,000 ya, where Neanderthals were already present, and probably remained until 50,000 ya [56]; this makes the prediction by Green et al. [27] probable.

\section{Future Directions}

\section{Increasing coverage, sequencing more Neanderthals, and the $\mathrm{Y}$ chromosome}

For a more comprehensive comparison of whole-genome sequences between Neanderthals and modern humans, the sequence coverage of Neanderthals has to be increased. The three approaches made so far to sequence the Neanderthal genome have resulted in the sequencing of only 65,000 bases [21], 1 million bases [19], and finally, the draft genome sequenced recently [27], consisting of only two-thirds of the whole genome with a mere $1.3 \times$ coverage. With such low coverage, it is hard to form meaningful contigs, and a number of important genetic entities will remain unnoticed. Even though it was beyond imagination to sequence a Pleistocene specimen a decade ago, the progress that has been made in the last 5 years is good enough to expect that more such specimens be sequenced in coming years. The more specimens from various geographic locations that are sequenced, the more likely it will be to construct a reference genome sequence for Neanderthals. As the human genome sequence varies considerably among different populations, it is expected that Neanderthals also have variation in their genomic sequences among different populations from different locations. Such variations can only be identified by sequencing a wide range of specimens, and these variations may again change insights into the Neanderthal-modern human relationship.

The sequencing of Neanderthals first started with its mitochondrial DNA in 1997 [25]. Comparisons between Neanderthal and modern human mitochondrial DNA have been made extensively, but these comparisons only reveal the maternally inherited difference between the species, as mitochondria DNA is transmitted maternally. The complete genomic sequence of Neanderthals published recently is also 
from a female specimen. Thus, the Y-chromosome of Neanderthals or paternal inheritance has yet to be examined. Comparisons of the Y chromosome sequence of Neanderthals with currently established Y-haplogroups for modern humans should provide some insights into the admixture hypothesis. With respect to the recent finding of admixture of Neanderthals with non-African populations, the Neanderthal $Y$ chromosome should not match the $Y$ haplogroups $\mathrm{A}$ or $\mathrm{B}$, as these haplogroups are the oldest of the clades and almost restricted to Africans and their descendants $[57,58]$. Since haplogroup $E$ is found in Africa, the Middle East, Southern Europe, and Asia [59-61], the Neanderthal Y chromosome may match this haplogroup, but it should not match the haplogroups E1bla*, E2b1, or B2ala, as they are specifically treated as Bantu expansion markers, while Neanderthals interbred only with nonAfricans [62, 63].

\section{Retrotransposon insertion polymorphism}

Almost half of the human genome comprises retrotransposons. Although they were overlooked for a significant period of time in our genetic study, their importance in chromosome structure, gene regulation, and disease predisposition has now been well established. Retroelements are widely divided into two categories-one with long terminal repeats (LTRs) and another without the LTRs. Short and long interspersed repeat elements (SINEs and LINEs, respectively) are two of the major classes of non-LTR retroelements, while SINEs are the more abundant class. Among SINEs, Alu is the predominant type of retroelement. Among all Alus found in the entire human genome, only about $0.5 \%$ is found to be present in the human genome but absent in orthologous regions of other primates and are thus identified as human-specific. This 'young' group of Alus is composed of only about 5,000 Alu elements that are believed to have integrated in the human genome after the divergence of humans and great apes [64-68]. Studying the retrotransposon insertion loci in Neanderthals will identify truly modern human-specific retrotransposon insertion polymorphisms. A similar comparative analysis would reveal other transposable elements, such as L1, SVAs, and HERVs, that are specific to modern humans only, as well as those that are specific to Neanderthals. Retroelements are particularly important in population genetics. It is extremely rare that a newly inserted transposable element is completely excised; thus, they act as a genetic fossil that is homoplasy-free. This identical-by-descent nature of retroelements makes them better markers for population and evolutionary studies than SNPs, in the sense that SNPs can, though rarely, be mutated back to the previous state. SNPs are also very hard to detect while handling ancient genomes due to transformation and deamination [69], while retrotransposon insertion polymorphisms (RIPs) refer to the presence or absence of a retrotransposon. Once a retrotransposon is inserted at a new location in an individual, it is subject to genetic drift. Over a short period, it starts spreading in to the population. Depending on when a retroelement has integrated at a certain locus, it will be shared by different species or, if recently enough, by different populations of the same species. Thus, RIPs occurring before the divergence of chimps and humans are shared by humans and chimps, but those occurring after are present only in humans. RIPs that are even more recent are specific to certain human populations only [70, 71]. For instance, some RIPs are found only in Africans, some in Han Chinese, and so on. The detailed information about all polymorphic retroelements and their frequency in different populations is extensively cataloged in the dbRIP database [43]. The identicalby-descent and homoplasy-free nature of RIPs makes them useful genetic markers in population and evolutionary genetics. The specificity of RIPs can play a significant role in answering the question of admixture of Neanderthals and modern humans. Finding RIPs that are shared between Neanderthals and non-African populations but not present in African populations can be considered solid support for the proposed admixture between Neanderthals and nonAfrican populations. In an ongoing study in our laboratory, over 500 RIPs were identified to be present in Khoisan and Bantu individuals, who represent the oldest lineage of modern humans from Southern Africa, but not in the reference human genome (unpublished). These oldest African lineage-specific RIPs theoretically should also be absent from Neanderthals.

\section{Human-specific unprocessed pseudogenes}

Pseudogenization has always been an interesting topic that has not been explored much. Many pseudogenes have been identified lately that lost their functional capacity in human lineage after the divergence of humans and chimps, particularly related to immunological functions [72]. The "less is more" hypothesis states that gene loss may direct evolutionary changes, as these pseudogenized genes have impacts on adaptation of the species through evolution [73]. Even though pseudogenization does not initiate under selective pressure, the gene loss is retained and subsequently allows adaptation. Pseudogenes are found particularly important in humans, and some of them have even been identified as being responsible for certain humanspecific phenotypes. For example, the sarcomeric myosin gene was lost at the time of emergence of the genus Homo and is thought to be responsible for marked reduction of hominin masticatory muscles, leading to expansion of brain 
size [74]. Analyzing pseudogenes in Neanderthals would be beneficial to identify genes that were lost after the divergence of Neanderthals and modern humans and determine what biological impact they may have.

\section{Human accelerated conserved noncoding sequences (HACNSs)}

HACNSs are sequences in the human genome that were conserved throughout vertebrate history but changed significantly after the divergence of humans and chimps. Human genomes have a number of such sequences that obtained surprisingly more mutations after the emergence of humans than before. These regions are typically rich in cis-regulatory transcriptional enhancers that render specific expression pattern of genes involved during development [75-77]. Identification of these cis-regulatory elements in human or other large genomes is done mostly by crossspecies sequence comparison, primarily because the functional cis-regulatory elements are generally unique to the genome, which makes paralogy-based identification of such sequences nearly impossible [78]. Since it has long been proposed that phenotypic variation between humans and chimps is mostly brought about by regulatory elements than coding sequences [79], it would be particularly interesting to make an elaborated comparison between differences in conserved regions in modern humans and Neanderthals. Such a comparison between the human genome and initial Neanderthal draft genome sequence [27], involving a total of 2,613 human accelerated regions, revealed that the Neanderthal sequence carried 3,259 human-specific changes in these regions. The comparison revealed that 51 positions in 45 regions were different between these two species; Neanderthals carried the ancestral form while all modern humans carry the human-specific variant. In a recent study, it has been found that the Neanderthal genome retains the ancestral state of a polymorphic site in a conserved noncoding microRNA, which is involved in regulating two genes that are important for teeth formation [80]. This may explain the dental differences between modern humans and Neanderthals. These findings are interesting enough to initiate further studies to analyze the probable impact of the variations in conserved noncoding sequences (CNSs) that exist between these two species. Furthermore, previously in three different studies, the number of HACNSs was found to be between 202 and 1175 [10, 11, 15]. All these studies used different methods to identify CNSs and included more species during the comparison. Their data can also be included in the future for the comparison of CNSs between modern humans and Neanderthals. The most rapidly evolving HACNS identified so far, named HACNS1, has accumulated 16 human-specific changes out of its 546 bases since the divergence of humans and chimps [12]. HACNS1 functions in multiple structures early in the developing stages of the mouse embryo as a transcriptional enhancer, including the developing anterior limb [12]. But, this function is missing for the orthologous enhancers in chimpanzee and rhesus, suggesting that HACNS1 has a different function in humans. When the chimpanzee enhancers are humanized by introducing 13 of the 16 human-specific substitutions, gene expression was observed in the limb, indicating that the substitutions that were identified by comparative analysis were directly responsible for the functional modification. In a recent study, 16 human-specific mutations in HACNS1 were also found in Neanderthals, suggesting that the phenotypic function that is related to this region was also expressed in Neanderthals [81]. The comparison can be extended to other human accelerated CNS regions to identify any substitutions that occurred after the divergence of humans from Neanderthals.

Conserved regions have also been presumed to play important roles in developing certain functions in humans for instance, noncoding regions of 150 presynaptic genes in humans are highly conserved and may have critical regulatory roles in the expression of these genes [82]. Comparative analyses of these regions between different populations of modern humans and Neanderthals may give directions to a better understanding of neurodevelopmental and psychiatric disorders. Involvement of noncoding sequences in the developing brain was also revealed by another experiment involving 49 human accelerated regions (HARs); the most accelerated region in the human genome, HAR1, is part of a noncoding RNA sequence expressed in the developing brain [83]. A study by Burbano et al. [83] identified that $8.3 \%$ of HAR substitutions are not shared between modern humans and Neanderthals, but the study did not focus on HAR1. A complete Neanderthal genomic sequence should provide an understanding of the evolutionary origin of these regulatory RNAs.

\section{Conclusion}

Despite many technical challenges, the whole-genome sequence for a Homo species other than modern human is now there for the first time in just five years of the establishment of the project. It is no longer a question of the possibility of sequencing more genomes from archaic remains, and more such achievements will only strengthen our understanding of human evolution. All of our knowledge on human species-specific genetic elements thus far is based on comparison with non-human primates, which gives us information only on how humans are different from other primates, such as chimpanzee-not how modern humans 
excelled from archaic humans. Modern humans are considerably superior to their predecessors, and the availability of high-quality Neanderthal sequences can shed some light on the genetic basis of this phenomenon. An initial comparison between the genome sequences of these two species has given us some valuable insight, and more studies are being conducted to identify further variations between Neanderthal and humans. However, a lot remains to be done, which has been discussed in this article, albeit briefly. Even though the sequence comparison itself can not provide much confidence on the biological differences between the Homo species, the application of reverse genetics can be initiated from this point to speculate and validate the possible biological effects of such sequence differences. Once the molecular functions of such differences are identified in an in vitro analysis, they can be administered into mouse models to observe the phenotypic results of these changes. One such experiment has already been implemented by administering a human version of FOXP2 in the mouse genome to observe its effects [84]. Many behavioral and qualitative traits of humans can be understood at the molecular level using similar approaches. There has also been hype by the media over resurrecting Neanderthals by modifying the chimpanzee genome to be more Neanderthal-like [85]. But, it is still an impossible task to regenerate a species just from the genomic sequence, even if the ethical issues can be resolved [86]. However, Neanderthals do not have to be reincarnated to provide us with an enormous opportunity in the field of human genetics and evolution, as their genome is already offering a lot to better our understanding of ourselves.

\section{Acknowledgments}

This work is in part supported by grants from the Canada Research Chair program, Canadian Foundation of Innovation (CFI), Ontario Ministry of Research \& Innovation (OMRI), Brock University, and Natural Sciences and Engineering Research Council (NSERC) to PL.

\section{References}

1. McDougall I, Brown FH, Fleagle JG. Stratigraphic placement and age of modern humans from Kibish, Ethiopia. Nature 2005;433:733-736.

2. Lu J, Ruhf ML, Perrimon N, Leder P. A genome-wide RNA interference screen identifies putative chromatin regulators essential for E2F repression. Proc Natl Acad Sci U S A 2007; 104:9381-9386.

3. Hublin JJ. Out of Africa: modern human origins special feature: the origin of Neandertals. Proc Natl Acad Sci U S A 2009; 106:16022-16027.
4. Stringer CB, Hublin J. New age estimates for the Swanscombe hominid, and their significance for human evolution. J Hum Evol 1999;37:873-877.

5. Finlayson C, Pacheco FG, Rodríguez-Vidal J, Fa DA, Gutierrez López JM, Santiago Pérez A, et al. Late survival of Neanderthals at the southernmost extreme of Europe. Nature 2006; 443:850-853.

6. Grun R, Stringer C, McDermott F, Nathan R, Porat N, Robertson $\mathrm{S}$, et al. U-series and ESR analyses of bones and teeth relating to the human burials from Skhul. J Hum Evol 2005;49:316-334.

7. Krause J, Orlando L, Serre D, Viola B, Prüfer K, Richards MP, et al. Neanderthals in central Asia and Siberia. Nature 2007;449:902-904.

8. Chimpanzee Sequencing and Analysis Consortium. Initial sequence of the chimpanzee genome and comparison with the human genome. Nature 2005;437:69-87.

9. Rhesus Macaque Genome Sequencing and Analysis Consortium, Gibbs RA, Rogers J, Katze MG, Bumgarner R, Weinstock GM, et al. Evolutionary and biomedical insights from the rhesus macaque genome. Science 2007;316:222-234.

10. Pollard KS, Salama SR, King B, Kern AD, Dreszer T, Katzman $\mathrm{S}$, et al. Forces shaping the fastest evolving regions in the human genome. PLoS Genet 2006;2:e168.

11. Prabhakar S, Noonan JP, Pääbo S, Rubin EM. Accelerated evolution of conserved noncoding sequences in humans. Science 2006;314:786.

12. Prabhakar S, Visel A, Akiyama JA, Shoukry M, Lewis KD, Holt A, et al. Human-specific gain of function in a developmental enhancer. Science 2008;321:1346-1350.

13. Haygood R, Fedrigo O, Hanson B, Yokoyama KD, Wray GA. Promoter regions of many neural- and nutrition-related genes have experienced positive selection during human evolution. Nat Genet 2007;39:1140-1144.

14. Clark AG, Glanowski S, Nielsen R, Thomas PD, Kejariwal A, Todd MA, et al. Inferring nonneutral evolution from human-chimp-mouse orthologous gene trios. Science 2003;302: 1960-1963.

15. Bird CP, Stranger BE, Liu M, Thomas DJ, Ingle CE, Beazley C, et al. Fast-evolving noncoding sequences in the human genome. Genome Biol 2007;8:R118.

16. Ponce de León MS, Golovanova L, Doronichev V, Romanova G, Akazawa T, Kondo O, et al. Neanderthal brain size at birth provides insights into the evolution of human life history. Proc Natl Acad Sci U S A 2008;105:13764-13768.

17. Mithen SJ. The Prehistory of the Mind: A Search for the Origins of Art, Religion and Science. Illustrated ed. London: Thames and Hudson, 1996.

18. Stringer C, Gamble C. In Search of the Neanderthals: Solving the Puzzle of Human Origins. London: Thames \& Hudson, 1993.

19. Green RE, Krause J, Ptak SE, Briggs AW, Ronan MT, Simons JF, et al. Analysis of one million base pairs of Neanderthal DNA. Nature 2006;444:330-336.

20. Green RE, Malaspinas AS, Krause J, Briggs AW, Johnson PL, Uhler C, et al. A complete Neandertal mitochondrial genome sequence determined by high-throughput sequencing. Cell 2008;134:416-426. 
21. Noonan JP, Coop G, Kudaravalli S, Smith D, Krause J, Alessi J, et al. Sequencing and analysis of Neanderthal genomic DNA. Science 2006;314:1113-1118.

22. Noonan JP, Hofreiter M, Smith D, Priest JR, Rohland N, Rabeder $\mathrm{G}$, et al. Genomic sequencing of Pleistocene cave bears. Science 2005;309:597-599.

23. Poinar HN, Schwarz C, Qi J, Shapiro B, Macphee RD, Buigues $\mathrm{B}$, et al. Metagenomics to paleogenomics: large-scale sequencing of mammoth DNA. Science 2006;311:392-394.

24. Ovchinnikov IV, Götherström A, Romanova GP, Kharitonov VM, Lidén K, Goodwin W. Molecular analysis of Neanderthal DNA from the northern Caucasus. Nature 2000;404:490-493.

25. Krings M, Stone A, Schmitz RW, Krainitzki H, Stoneking M, Pääbo S. Neandertal DNA sequences and the origin of modern humans. Cell 1997;90:19-30.

26. Briggs AW, Good JM, Green RE, Krause J, Maricic T, Stenzel U, et al. Targeted retrieval and analysis of five Neandertal mtDNA genomes. Science 2009;325:318-321.

27. Green RE, Krause J, Briggs AW, Maricic T, Stenzel U, Kircher $\mathrm{M}$, et al. A draft sequence of the Neandertal genome. Science 2010;328:710-722.

28. Gravina B, Mellars P, Ramsey CB. Radiocarbon dating of interstratified Neanderthal and early modern human occupations at the Chatelperronian type-site. Nature 2005;438:51-56.

29. Mellars P, Gravina B, Bronk Ramsey C. Confirmation of Neanderthal/modern human interstratification at the Chatelperronian type-site. Proc Natl Acad Sci U S A 2007;104: 3657-3662.

30. Zilhão J, d'Errico F, Bordes JG, Lenoble A, Texier JP, Rigaud JP. Analysis of Aurignacian interstratification at the Chatelperronian-type site and implications for the behavioral modernity of Neandertals. Proc Natl Acad Sci U S A 2006;103:1264312648.

31. Trinkaus E, Moldovan O, Milota S, Bîlgăr A, Sarcina L, Athreya $\mathrm{S}$, et al. An early modern human from the Pestera cu Oase, Romania. Proc Natl Acad Sci U S A 2003;100:11231-11236.

32. Bailey SE, Weaver TD, Hublin JJ. Who made the Aurignacian and other early Upper Paleolithic industries? J Hum Evol 2009;57:11-26.

33. Currat M, Excoffier L. Modern humans did not admix with Neanderthals during their range expansion into Europe. PLoS Biol 2004;2:e421.

34. Evans PD, Mekel-Bobrov N, Vallender EJ, Hudson RR, Lahn $\mathrm{BT}$. Evidence that the adaptive allele of the brain size gene microcephalin introgressed into Homo sapiens from an archaic Homo lineage. Proc Natl Acad Sci U S A 2006;103:18178-18183.

35. Wall JD, Hammer MF. Archaic admixture in the human genome. Curr Opin Genet Dev 2006;16:606-610.

36. Krause J, Lalueza-Fox C, Orlando L, Enard W, Green RE, Burbano HA, et al. The derived FOXP2 variant of modern humans was shared with Neandertals. Curr Biol 2007;17:19081912.

37. Boeke JD, Pickeral OK. Retroshuffling the genomic deck. Nature 1999;398:108-109, 111.

38. Damert A, Raiz J, Horn AV, Löwer J, Wang H, Xing J, et al. 5'-Transducing SVA retrotransposon groups spread efficiently throughout the human genome. Genome Res 2009;19:1992-
2008.

39. Hancks DC, Ewing AD, Chen JE, Tokunaga K, Kazazian HHJr. Exon-trapping mediated by the human retrotransposon SVA. Genome Res 2009;19:1983-1991.

40. Cordaux R, Batzer MA. The impact of retrotransposons on human genome evolution. Nat Rev Genet 2009;10:691-703.

41. Kazazian HH Jr. Mobile elements: drivers of genome evolution. Science 2004;303:1626-1632.

42. Wang J, Song L, Gonder MK, Azrak S, Ray DA, Batzer MA, et al. Whole genome computational comparative genomics: A fruitful approach for ascertaining Alu insertion polymorphisms. Gene 2006;365:11-20.

43. Wang J, Song L, Grover D, Azrak S, Batzer MA, Liang P. dbRIP: a highly integrated database of retrotransposon insertion polymorphisms in humans. Hum Mutat 2006;27:323-329.

44. Callinan PA, Batzer MA. Retrotransposable elements and human disease. Genome Dyn 2006;1:104-115.

45. Orlando L, Darlu P, Toussaint M, Bonjean D, Otte M, Hänni C. Revisiting Neandertal diversity with a 100,000 year old mtDNA sequence. Curr Biol 2006;16:R400-R402.

46. Serre D, Langaney A, Chech M, Teschler-Nicola M, Paunovic $\mathrm{M}$, Mennecier $\mathrm{P}$, et al. No evidence of Neandertal mtDNA contribution to early modern humans. PLoS Biol 2004;2:E57.

47. Lalueza-Fox C, Römpler H, Caramelli D, Stäubert C, Catalano $\mathrm{G}$, Hughes D, et al. A melanocortin 1 receptor allele suggests varying pigmentation among Neanderthals. Science 2007;318: 1453-1455.

48. Lalueza-Fox C, Gigli E, de la Rasilla M, Fortea J, Rosas A, Bertranpetit J, et al. Genetic characterization of the ABO blood group in Neandertals. BMC Evol Biol 2008;8:342.

49. Lalueza-Fox C, Gigli E, de la Rasilla M, Fortea J, Rosas A. Bitter taste perception in Neanderthals through the analysis of the TAS2R38 gene. Biol Lett 2009;5:809-811.

50. Noonan JP. Neanderthal genomics and the evolution of modern humans. Genome Res 2010;20:547-553.

51. Morin RD, O'Connor MD, Griffith M, Kuchenbauer F, Delaney A, Prabhu AL, et al. Application of massively parallel sequencing to microRNA profiling and discovery in human embryonic stem cells. Genome Res 2008;18:610-621.

52. Parikh H, Lyssenko V, Groop LC. Prioritizing genes for follow-up from genome wide association studies using information on gene expression in tissues relevant for type 2 diabetes mellitus. BMC Med Genomics 2009;2:72.

53. Hodgson JA, Disotell TR. No evidence of a Neanderthal contribution to modern human diversity. Genome Biol 2008;9:206.

54. Hardy J, Pittman A, Myers A, Gwinn-Hardy K, Fung HC, de Silva $\mathrm{R}$, et al. Evidence suggesting that Homo neanderthalensis contributed the H2 MAPT haplotype to Homo sapiens. Biochem Soc Trans 2005;33(Pt 4):582-585.

55. Lari M, Rizzi E, Milani L, Corti G, Balsamo C, Vai S, et al. The microcephalin ancestral allele in a Neanderthal individual. PLoS One 2010;5:e10648.

56. Akazawa T, Aoki K, Bar-Yosef O. Neandertals and Modern Humans in Western Asia. New York: Springer, 1999.

57. Semino O, Santachiara-Benerecetti AS, Falaschi F, CavalliSforza LL, Underhill PA. Ethiopians and Khoisan share the deepest clades of the human Y-chromosome phylogeny. Am J 
Hum Genet 2002;70:265-268.

58. Underhill PA, Passarino G, Lin AA, Shen P, Mirazón Lahr M, Foley RA, et al. The phylogeography of $Y$ chromosome binary haplotypes and the origins of modern human populations. Ann Hum Genet 2001;65(Pt 1):43-62.

59. Semino O, Magri C, Benuzzi G, Lin AA, Al-Zahery N, Battaglia $\mathrm{V}$, et al. Origin, diffusion, and differentiation of Y-chromosome haplogroups $\mathrm{E}$ and $\mathrm{J}$ : inferences on the neolithization of Europe and later migratory events in the Mediterranean area. Am J Hum Genet 2004;74:1023-1034.

60. Karafet TM, Mendez FL, Meilerman MB, Underhill PA, Zegura SL, Hammer MF. New binary polymorphisms reshape and increase resolution of the human Y chromosomal haplogroup tree. Genome Res 2008;18:830-838.

61. Cruciani F, Santolamazza P, Shen P, Macaulay V, Moral P, Olckers A, et al. A back migration from Asia to sub-Saharan Africa is supported by high-resolution analysis of human Y-chromosome haplotypes. Am J Hum Genet 2002;70:11971214.

62. Beleza S, Gusmão L, Lopes A, Alves C, Gomes I, Giouzeli M, et al. Micro-phylogeographic and demographic history of Portuguese male lineages. Ann Hum Genet 2006;70(Pt 2): 181-194.

63. Berniell-Lee G, Calafell F, Bosch E, Heyer E, Sica L, Mouguiama-Daouda P, et al. Genetic and demographic implications of the Bantu expansion: insights from human paternal lineages. Mol Biol Evol 2009;26:1581-1589.

64. Batzer MA, Deininger PL. A human-specific subfamily of Alu sequences. Genomics 1991;9:481-487.

65. Batzer MA, Rubin CM, Hellmann-Blumberg U, AlegriaHartman M, Leeflang EP, Stern JD, et al. Dispersion and insertion polymorphism in two small subfamilies of recently amplified human Alu repeats. J Mol Biol 1995;247:418-427.

66. Matera AG, Hellmann U, Schmid CW. A transpositionally and transcriptionally competent Alu subfamily. Mol Cell Biol 1990;10:5424-5432.

67. Roy-Engel AM, Carroll ML, Vogel E, Garber RK, Nguyen SV, Salem AH, et al. Alu insertion polymorphisms for the study of human genomic diversity. Genetics 2001;159:279-290.

68. Roy AM, Carroll ML, Kass DH, Nguyen SV, Salem AH, Batzer MA, et al. Recently integrated human Alu repeats: finding needles in the haystack. Genetica 1999;107:149-161.

69. Briggs AW, Stenzel U, Johnson PL, Green RE, Kelso J, Prüfer K, et al. Patterns of damage in genomic DNA sequences from a Neandertal. Proc Natl Acad Sci U S A 2007;104:14616-14621.

70. Mills RE, Bennett EA, Iskow RC, Devine SE. Which transposable elements are active in the human genome? Trends Genet 2007;23:183-191.
71. Wang X, Grus WE, Zhang J. Gene losses during human origins. PLOS Biol 2006; 4:e52.

72. Olson MV. When less is more: gene loss as an engine of evolutionary change. Am J Hum Genet 1999;64:18-23.

73. Stedman HH, Kozyak BW, Nelson A, Thesier DM, Su LT, Low DW, et al. Myosin gene mutation correlates with anatomical changes in the human lineage. Nature 2004;428:415-418.

74. Pennacchio LA, Ahituv N, Moses AM, Prabhakar S, Nobrega MA, Shoukry M, et al. In vivo enhancer analysis of human conserved non-coding sequences. Nature 2006;444:499-502.

75. Nobrega MA, Ovcharenko I, Afzal V, Rubin EM. Scanning human gene deserts for long-range enhancers. Science 2003; 302:413.

76. Boffelli D, Nobrega MA, Rubin EM. Comparative genomics at the vertebrate extremes. Nat Rev Genet 2004;5:456-465.

77. Bejerano G, Haussler D, Blanchette M. Into the heart of darkness: large-scale clustering of human non-coding DNA. Bioinformatics 2004;20 Suppl 1:i40-i48.

78. King MC, Wilson AC. Evolution at two levels in humans and chimpanzees. Science 1975;188:107-116.

79. Lopez-Valenzuela M, Ramírez O, Rosas A, García-Vargas S, de la Rasilla M, Lalueza-Fox C, et al. An ancestral miR-1304 allele present in Neanderthals regulates genes involved in enamel formation and could explain dental differences with modern humans. Mol Biol Evol 2012;29:1797-1806.

80. Hünemeier T, Ruiz-Linares A, Silveira A, Paixão-Côrtes VR, Salzano FM, Bortolini MC. Brief communication: Population data support the adaptive nature of HACNS1 sapiens/neandertal-chimpanzee differences in a limb expression domain. Am J Phys Anthropol 2010;143:478-481.

81. Hadley D, Murphy T, Valladares O, Hannenhalli S, Ungar L, Kim J, et al. Patterns of sequence conservation in presynaptic neural genes. Genome Biol 2006;7:R105.

82. Pollard KS, Salama SR, Lambert N, Lambot MA, Coppens S, Pedersen JS, et al. An RNA gene expressed during cortical development evolved rapidly in humans. Nature 2006;443: 167-172.

83. Burbano HA, Green RE, Maricic T, Lalueza-Fox C, de la Rasilla M, Rosas A, et al. Analysis of human accelerated DNA regions using archaic hominin genomes. PLoS One 2012;7:e32877.

84. Enard W, Gehre S, Hammerschmidt K, Hölter SM, Blass T, Somel M, et al. A humanized version of FOXP2 affects cortico-basal ganglia circuits in mice. Cell 2009;137:961-971.

85. Wade N. Scientists in germany draft Neanderthal genome. The New York Times 2009 Feb 12:A12.

86. Pennisi E. Paleogenetics. Cloned Neandertals still in the realm of sci-fi. Science 2010;328:682-683. 